J. AMER. Soc. HORT. SCI. 115(1):153-157. 1990.

\title{
Characterization of sugary-1 (su-1) sugary enhancer (se) Kernels in Segregating Sweet Corn Populations
}

\author{
Don R. La Bonte ${ }^{1}$ and John A. Juvik ${ }^{2}$ \\ Department of Horticulture, University of Illinois, Urbana, IL 61801 \\ Additional index words. maize, Zea rnays, endosperm mutation, vegetable breeding, quality, carbohydrates, carotene
}

\begin{abstract}
A single-kernel, sugar analysis technique was used to study the genetic relationship between morphological and metabolic traits previously associated with expression of the sugary enhancer (se) endosperm mutation in a su-1 sweet corn (Zea mays L.) background. Analysis of sucrose and total carotene content in su-1 kernel populations segregating for se showed that light-yellow kernel color was a reliable phenotypic indicator for kernels homozygous for the $s e$ gene. High levels of kernel maltose was not always indicative of $s u-1$ se kernels in mature (55 days after pollination) kernel populations. Characteristic high levels of percent moisture in su-1 se kernels at 28 and 35 days post-pollination were identified as an expression of high sugar content. Kernels homozygous for su-1 se were also found to weigh less at maturity than su-1 Se kernels, and se was found to be partially expressed in a heterozygous condition.
\end{abstract}

Traditional sweet corn cultivars have the endosperm carbohydrate fraction modified by the sugary-1 (su-1) mutation. Although kernels of $s u-1$ cultivars have a very desirable, creamy texture at eating maturity, they contain about half the total sugar content of "super sweet" cultivars (Cameron and Teas, 1954; Creech, 1965; Creech, 1968; Laughnan, 1953). Endosperm carbohydrates of super sweet cultivars are modified by mutations such as shrunken-2 (sh-2,), brittle (bt), and brittle-2 (bt-2), or the combination of the mutants amylose extender (se), dull (du), and waxy (wx) (Creech, 1968). In addition to consumer preference for the higher sugar levels, improved postharvest storage properties are also associated with high sugar content (Garwood et al., 1976; Flora and Wiley, 1974).

Super sweet cultivars retain higher levels of sugar and moisture for a longer period after harvest than traditional $S U-1$ cultivars (Garwood et al., 1976; Soberlaske and Andrew, 1978; Warm et al., 1971). One disadvantage of super sweet cultivars is that the texture is not as creamy as that found in $s u-1$ cultivars (Gonzales et al., 1974, 1976). Kernel creaminess depends on high levels of phytoglycogen, a water-soluble starch (Gonzales et al., 1976).

The sugary enhancer (se) gene is a recessive modifier of the su-1 endosperm mutation (Ferguson et al., 1978). When homozygous, the se allele increases total sugar levels in $s u-1$ kernels to levels comparable to those in $s h-2$ kernels without a reduction in phytoglycogen (Gonzales et al., 1974; Gonzales et al., 1976). Hence, su-1 se kernels contain the desirable textural characteristics of $s u-1$ kernels and the high levels of sugar found in super sweet cultivars. Desirable moisture and sugar retention during postharvest storage are also associated with su-1 se kernels (Carey et al., 1982; Gonzales et al., 1976).

\footnotetext{
Received for publication 9 Jan. 1989. We thank Joseph G. Sullivan for his advice and aid in the statistical analysis of the data and Robert K. Proksch for technical assistance. This research was funded by a grant from the American Seed Research Foundation, Washington, D.C., United States-Israel Binational Agricultural and Research Development (BARD) grant no. US-1213-86, and by project $65-0330$ of the Agricultural Experiment Station, Univ. of Illinois, Urbana-Champaign. The cost of publishing this paper was defrayed in part by the payment of page charges. Under postal regulations, this paper therefore must be hereby marked advertisement solely to indicate this fact.

'Former graduate research assistant, Univ. of Illinois. Currently Assistant Professor, Dept. of Horticulture, Louisiana State Univ., Baton Rouge, LA 70803. ${ }^{2}$ Associate Professor.
}

Unlike other endosperm mutations, $s u-1$ kernels homozygous for $s e$ are not always phenotypically different from $s u-1 \mathrm{Se}$ kernels in segregating populations. Two phenotypic traits, lighter yellow kernel color and slower dry-down (Gonzales et al., 1974), are associated with kernels homozygous for se, but we have observed that these traits are variable in expression, depending on the genetic background and kernel maturity. This lack of a readily identifiable trait has limited breeders' ability to incorporate se into elite germplasm.

Our objective was to more fully develop the relationship between metabolic and morphological traits associated with se gene expression, and to determine whether these traits facilitate identification of individual su-1 se kernels in segregating populations.

\section{Materials and Methods}

Plant materials. Three inbred maize lines IL451b (su-1 Se) IL678a (su-1 Se), and IL677a (su-1 se) developed at the Univ. of Illinois Agricultural Experiment Station were used in this study. Both su-1 Se inbreds were crossed with IL677a to generate $F_{1}$ seed using IL677a as the female. $F_{1}$ seed was planted and selfed to generate $\mathrm{F}_{2}$ seed. Rows of 25 seeds of each of the inbreds and hybrids were planted on the Dept. of Horticulture's Vegetable Research Farm in South Urbana in a randomized block design with four replicates. Six or seven ears in each row were self-pollinated, bagged to prevent contamination, and dated. An additional six or seven ears of IL677a in each replicate were pollinated with pollen from IL678a to create $F_{1}$ seed. Ears were harvested at 21 (eating maturity), 28, 35, and 42 days after pollination (DAP), immediately frozen in liquid $\mathrm{N}_{2}$ and then stored in a freezer at $-80 \mathrm{C}$ for later analysis. Additional ears from each replicate were harvested at $55 \mathrm{DAP}$, dried in a forcedair oven at $33 \mathrm{C}$ for $72 \mathrm{hr}$, and stored in the laboratory as maturedry kernel samples.

Extraction and analysis of maize kernels. Individual kernels were removed from the frozen immature ears, weighed, freezedried, reweighed, placed in 1.5-ml plastic microcentrifuge tubes, and ground into a powder with a Phillips screwdriver. Mature dry kernels were weighed and ground in a rotary mill with a 20 -mesh screen. Ground powder (100 to $200 \mathrm{mg}$ ) from a mature-dry kernel or the entire freeze-dried kernel was placed in a microcentrifuge tube and extracted four times with $1.0-\mathrm{ml}$ por- 
tions of $95 \%$ ethanol on a rotary-evaporator at $70 \mathrm{C}$ for $15 \mathrm{~min}$ per extraction. After each extraction, the vials were centrifuged for $10 \mathrm{~min}$ at $8000 \times \mathrm{g}$. The supernatant fluids were brought up to a $5.0-\mathrm{ml}$ volume with ethanol.

The sugars contained within a 50- $\mu$ l aliquot of each extract were converted to trimethylsilyl (TMS) derivatives in the sample after their stabilization as oximes. Juvik and La Bonte (1988) described in detail the derivatization technique and quantification of sugars using capillary gas chromatography.

Adsorption of total carotene in ethanol extracts was determined using a Zeiss PMQ II spectrophotometer. Adsorption values were converted to a dry-weight basis (mg total carotene/ g dry kernel weight) by comparison with a set of Sudan I (phenylazo-2-napthol) 95\% ethanol standards. Quantification is based on $0.04 \mathrm{~mm}$ of Sudan I $=2.35 \mathrm{mg}$ total carotene/liter at $436 \mathrm{~nm}$ in an acetone : isopropynol solvent (Association of Official Analytical Chemists, 1984). The exchange of ethanol for acetone : isopropynol did not significantly $(r=0.997)$ alter the slope of the standard solution set. A typical adsorption spectra between 400 and $450 \mathrm{~nm}$ for carotenoids (Davies, 1976) was observed (La Bonte, 1988).

Effect of se gene expression on total carotene content and sucrose (Expt. 1). An experiment was conducted to determine the extent of variation in total kernel carotene content between $S U-1 \mathrm{Se}$ inbreds and a $S U-1$ se inbred, between ears of the same inbred, and between kernels from the same ear. A selfed ear from each of the four replicates of the inbreds IL677a (se), IL451b ( $\mathrm{Se}$ ), and IL678a ( $\mathrm{Se}$ ) at 21 DAP were used. Six kernels were selected from each ear for single-kernel analysis. The total sums of squares from an analysis of variance (ANOVA) was partitioned into component sums of squares for genotype, earto-ear within a genotype, and kernel-to-kernel within an ear. Means and SDS for total carotene represents a sum of all kernels for each inbred.

To determine the relationship between se gene expression and kernel color, 95 kernels were randomly selected from a single ear of the $\mathrm{F}_{2}$ kernel population: (IL677a $\times$ IL678a) self, for single-kernel analysis at 21 DAP. Individual kernels were classified visually as either light or dark yellow before extraction and quantification of sucrose and total carotene. These two kernel classes were subjected to $X^{2}$ analysis to determine whether expected segregation ratios would be obtained. Means were separated by the $\mathrm{F}$ test. A multivariant analysis was used to solve a quadratic equation with total carotene and sucrose as variables. The solutions from the quadratic equation were then used to develop $95 \%$ confidence ellipses.

To determine whether endosperm heterozygous for these gene affects endosperm sucrose, 25 kernels were randomly selected from individual ears harvested at 21 DAP of IL678a, IL677a, and their $F_{1}($ IL677a $\times$ IL678a), for single-kernel analysis. The triploid maize endosperm arises from the union of two polar nuclei contributed by the female and one nucleus contributed by the male. Dosage for se varies from zero for IL678a, two for $F_{1}$ kernels (IL677a as female), to three for IL677a. Data were subjected to ANOVA and means were separated by Fisher's LSD.

Effect of se gene expression on kernel maltose and weight (Expt. 2). To determine the relationship between se gene expression, mature kernel dry weight, and maltose level, 50 kernels from a single ear were randomly selected from the mature dry $\mathrm{F}_{2}$ kernel population: (IL451b $\times$ IL677a) self, for single-kernel analysis. Individual kernels were classified visually as either light or dark-yellow. Kernel dry weight represents the weight of the whole kernel after drying in a forced-air oven at 33C for $72 \mathrm{hr}$. Data were subjected to ANOVA and means were separated by the $\mathrm{F}$ test. Development of $95 \%$ confidence ellipses, used in the study concerning kernel dry weight, was described previously.

Effect of se gene expression on percent moisture (Expt. 3). To determine the relationship between se gene expression and percent moisture, 151 kernels were randomly selected from an $\mathrm{F}_{2}$ kernel population from a single ear of (IL677a $\times$ IL678a) self harvested at 35 DAP for single-kernel sugar analysis. Differences in percent moisture content between $s u-1$ se and $s u-1 \mathrm{Se}$ kernels are greatest at 35 and 42 DAP (La Bonte, 1988). Percent moisture is a measure of the proportion of the total kernel weight due to water content, and is calculated from the following equation: percent moisture content $=($ fresh kernel weight - dry kernel weighed/fresh weight $\times 100$. Data were subjected to regression analysis at $P=0.05$, and means were separated by the $\mathrm{F}$ test.

\section{Results and Discussion}

Carotene and sucrose contents. Kernels of the su- 1 se inbred, IL677a, possessed a significantly lower amount of total carotene than kernels of the su-1 Se inbreds, IL451b and IL678a (Table 1). The primary source of the total variability in kernel total carotene comes from genetic differences between the two inbreds in each comparison. Sources of variation due to ears-withininbreds and kernels-within-ears accounted for a smaller proportion of the total variation in total carotene. Only ear-to-ear variation within inbreds for the comparison involving kernels of IL677a and IL678a was significantly different.

The proportion of variation in total carotene attributed to the genotype of the kernel is similar to that observed for sucrose in a previous comparison of IL677a and IL45lb (Juvik and La Bonte, 1988). Together, these results indicate that kernels homozygous for se might be identified by single-kernel analysis in segregating plant populations based on total carotene content and sucrose, particularly when segregation is occurring on an individual ear.

The 95 kernels selected from an $\mathrm{F}_{2}$ kernel population at 21 DAP fit a significant $\mathrm{X}^{2}$ distribution $\left(\mathrm{X}^{2}=0.032,0.50>P>\right.$ 0.20 ) for a 3:1 segregation ratio for se (3 Se: 1 se se) based on kernel color (Table 2). The light-yellow kernel class had 63\% less total carotene than the dark-yellow kernel class. These data

Table 1. Total carotene in inbred kernels of IL677a, IL451b, and IL678a with relative contribution of each source of variation to the total variation in total carotene 21 days after pollination.

\begin{tabular}{|c|c|c|c|c|}
\hline \multirow{2}{*}{\multicolumn{2}{|c|}{$\begin{array}{l}\text { Mean total } \\
\text { carotene concn } \\
\left(\mathrm{mg} \cdot \mathrm{g}^{-1} \text { dry wt) and SDS }\right.\end{array}$}} & \multicolumn{3}{|c|}{$\begin{array}{c}\text { Sources of variation } \\
(\% \text { of total })^{z} \\
\text { and their level of } \\
\text { significance }\end{array}$} \\
\hline & & Inbreds & $\begin{array}{c}\text { Ears } \\
\text { (inbreds) }\end{array}$ & $\begin{array}{c}\text { Kernels } \\
\text { (ears) }\end{array}$ \\
\hline IL677a & IL451b & & & \\
\hline $\begin{array}{c}0.244 \quad 0.056 \\
\text { IL677a }\end{array}$ & $\begin{array}{c}0.404 \quad 0.096 \\
\text { IL678a }\end{array}$ & $50^{*}$ & $17^{*}$ & $8^{\mathrm{NS}}$ \\
\hline $0.244 \quad 0.056$ & $0.445 \quad 0.060$ & $74^{*}$ & $4^{\mathrm{NS}}$ & $6^{\mathrm{NS}}$ \\
\hline
\end{tabular}

${ }^{2}$ Percent of total variation uniquely due to genotype (inbred), ears within inbreds, and kernels within ears.

YLevel of significance for inbreds, ears within inbreds, and kernels within ears are presented as NS when $P>0.05$, and as ${ }^{*}$ when $P<$ 0.001 . 
Table 2. Chemical variation in individual kernels visually classified as light (L) or dark (D) yellow from $\mathrm{F}_{2}$ su-1 kernel populations segregating for the se gene. ${ }^{\mathrm{z}}$

\begin{tabular}{|c|c|c|c|c|c|c|c|c|c|c|c|c|}
\hline \multirow[b]{2}{*}{ Kernel maturity } & \multirow{2}{*}{$\begin{array}{c}\mathrm{F}_{2} \\
\text { pedigree }\end{array}$} & \multirow{2}{*}{$\begin{array}{c}\text { No. } \\
\text { kernels }\end{array}$} & \multicolumn{2}{|c|}{$\begin{array}{c}\text { Total } \\
\text { carotene } \\
\left(\mathrm{mg} \cdot \mathrm{g}^{-1}\right)\end{array}$} & \multicolumn{2}{|c|}{$\begin{array}{l}\text { Sucrose } \\
\left(\mathrm{mg} \cdot \mathrm{g}^{-1}\right. \\
\text { dry wt) }\end{array}$} & \multicolumn{2}{|c|}{$\begin{array}{l}\text { Maltose } \\
\text { (mg } \mathrm{g}^{-1} \\
\text { dry wt) }\end{array}$} & \multicolumn{2}{|c|}{$\begin{array}{c}\text { Kernel } \\
\text { dry } \\
\text { wt (g) }\end{array}$} & \multicolumn{2}{|c|}{$\begin{array}{c}\text { Moisture } \\
\text { content } \\
(\%) \\
\end{array}$} \\
\hline & & & $\mathrm{L}$ & $\mathrm{D}$ & $\mathrm{L}$ & $\mathrm{D}$ & $\mathrm{L}$ & $\mathrm{D}$ & $\mathrm{L}$ & $\mathrm{D}$ & $\mathrm{L}$ & $\mathrm{D}$ \\
\hline $\begin{array}{l}35 \text { DAP } \\
\text { Mature-dry seed }\end{array}$ & $7 a \times U 151 b$ & $\begin{array}{r}95 \\
151 \\
50\end{array}$ & & $\begin{array}{c}0.281 \\
0.246 \\
\text { NA }\end{array}$ & $\begin{array}{l}71^{*} \\
37^{*} \\
48^{*}\end{array}$ & $\begin{array}{l}46 \\
24 \\
33\end{array}$ & $\begin{array}{l}3.7^{*} \\
9.4^{*} \\
0.71^{*}\end{array}$ & $\begin{array}{l}2.1 \\
5.7 \\
.05\end{array}$ & $\begin{array}{c}\text { NA } \\
\text { NA } \\
0.20^{*}\end{array}$ & $\begin{array}{l}\text { NA } \\
0.25\end{array}$ & $\begin{array}{l}72^{*} \\
62^{*} \\
\text { NA }\end{array}$ & $\begin{array}{r}70 \\
59 \\
\text { NA }\end{array}$ \\
\hline
\end{tabular}

${ }^{2}$ Means with an asterisk represent paired comparisons between light and dark-yellow kernels significantly different at $P<0.05$.

$\mathrm{YDAP}=$ days after pollination.

substantiate the use of subjective visual color determination as a means of selection for variation in endosperm carotene content. These same two kernel classes differed significantly in sucrose content. Light-yellow kernels contained $65 \%$ more sucrose than dark kernels (Table 2).

These results are further exemplified in $95 \%$ confidence ellipses that divide the population into two kernel classes based on sucrose and total carotene (Fig. 1). One class contains kernels within relatively low levels of sucrose and high levels of total carotene. These kernels were visually identified as dark yellow and likely are homozygous or heterozygous for the partially dominant $S e$ allele. The other class contains kernels that are high in sucrose and low in total carotene. These kernels were identified as light yellow and are likely homozygous for $s e$. The overlap between the confidence intervals produces a third class of kernels, intermediate in sucrose. The intermediate level of sucrose may represent expression of se in a heterozygous condition. Previous work suggested $S e$ is not completely

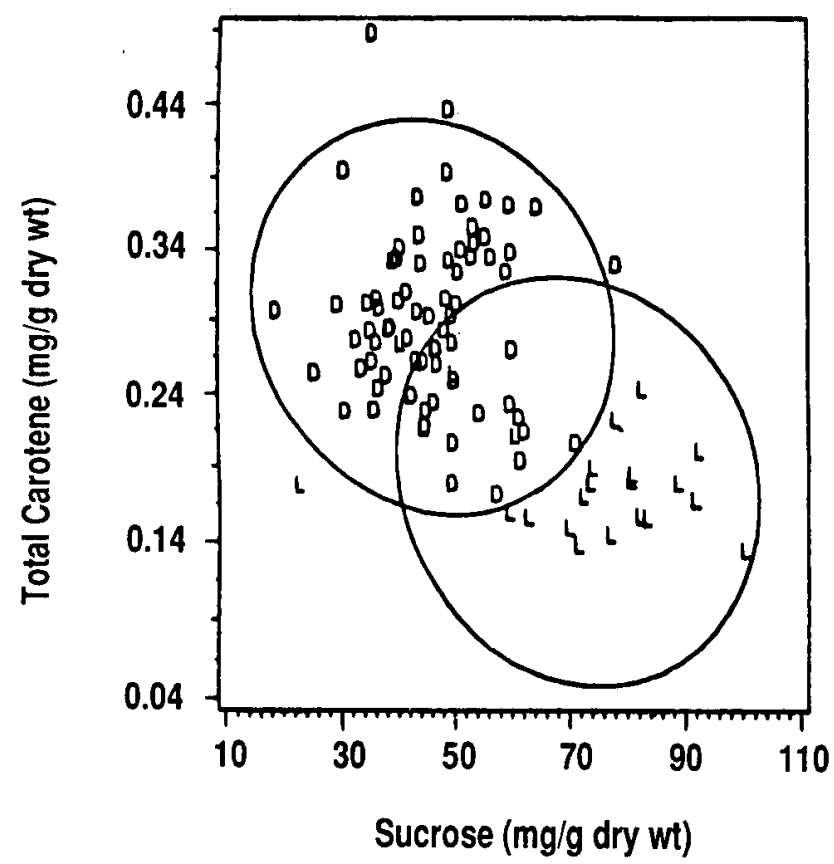

Fig. 1. Distribution of individual kernels at 21 days after pollination with respect to total carotene and sucrose from the $F_{2}$ population: (IL677a $\times$ IL451b)self, segregating for the se gene. Ellipses represent 95\% confidence intervals. $\mathrm{L}=$ light-yellow kernels $\mathrm{D}=$ darkyellow kernels. dominant over se (Ferguson et al., 1979; La Bonte and Juvik, 1987a). Expression of $s e$ in a heterozygous condition is clearly demonstrated in a comparison of kernels with varying doses of $s e$ in the endosperm (Table 3). $\mathrm{F}_{1}$ kernels (se se $\mathrm{Se}$ ) are significantly higher in sucrose than kernels of the inbred IL678a ( $\mathrm{Se}$ $\mathrm{Se} \mathrm{Se}$ ) and significantly lower than the inbred IL677a (se se se).

Several kernels (Fig. 1) fall outside the ellipsoidal intervals. One kernel is low in both total carotene (dark-yellow) and sucrose. These kernels may simply represent either random outliers or recombinant types in which a crossover may have occurred between the se gene and a gene affecting carotene production. The latter hypothesis is a possibility because the biochemical pathways producing carotenoids and carbohydrates are distinct. Various white endosperm mutants in maize have enzymatic deficiencies in pathways leading from precursor molecules to cyclic carotenoids (Robertson et al., 1978). Cyclic carotenoids, such as ( $\beta$-zeacarotene and $\alpha$-carotene, are the principal carotenoids found in frozen yellow maize endosperm (Lee et al., 1981). In contrast, endosperm carbohydrate mutations in maize consist primarily of enzymatic deficiencies involved in the formation of starch and phytoglycogen from mono- and disaccharides (Shannon and Garwood, 1984).

A nondestructive method is proposed as a means of determining the appropriate hypothesis explaining the relationship between se and kernel color. Embryos can be excised from kernels and cultured (Green and Phillips, 1975) on modified (low-salt) Linsmaier and Skoog basal medium (Linsmaier and Skoog, 1965). The sucrose and total carotene in the excised endosperm can be quantified. Seedlings from developing embryos can then be transplanted and grown to maturity. Singlekernel analysis of selfed seed from these transplants would indicate the presence or absence of $s e$. The development of a darkyellow $S U-1$ se stock would confirm the linkage hypothesis. Such a stock would be desirable to the sweet corn processing

Table 3. Sucrose content for inbreds and $F_{1}$ kernels (25 for each genotype) from individual ears containing various doses of se in the endosperm at 21 days after pollination.

\begin{tabular}{lcc}
\hline Genotype & $\begin{array}{c}\text { Gene } \\
\text { dosage in } \\
\text { endosperm }\end{array}$ & $\begin{array}{c}\text { Mean sucrose } \\
\text { concn } \\
\left(\mathrm{mg} \cdot \mathrm{g}^{-1} \mathrm{dry} \mathrm{wt}\right)^{z}\end{array}$ \\
\hline IL677a & se se se & $76 \mathrm{a}$ \\
IL677a $\times$ IL678a & se se Se & $71 \mathrm{~b}$ \\
IL678a & Se Se Se & $47 \mathrm{c}$ \\
\hline
\end{tabular}

${ }^{2}$ Means followed by the same letter are not significantly different based on LSD test at $P=0.05$. 
industry in the light of consumer preference for dark-yellow kernels of canned or frozen corn.

Kernel maltose and dry weight. Significantly more maltose was observed in mature-dry, light-yellow $\mathrm{F}_{2}$ kernels than in dark-yellow kernels (Table 2). As in the previous experiment, light-yellow kernels also contained significantly higher levels of sucrose. Although most individual kernels followed these same trends for sucrose and maltose (Fig. 2), there were some light-yellow kernels that contained high levels of sucrose, even in comparison to other light-yellow kernels, but no detectable level of maltose. This observation and previous data (Carey et al., 1982) suggests that higher concentrations of maltose may not always be found in se kernels. Several hypotheses explain the presence of high levels of sucrose and low levels of maltose. First, if elevated maltose is a pleiotrophic expression of $s e$, this amplification may be better observed in kernels at 28 or 35 DAP and not in mature-dry kernels. Maltose content in mature-dry kernels may be more sensitive to environmental variations since levels of this compound in kernels of IL677a have been found to vary dramatically from one year to the next (Carey et al., 1982). Second, segregation of other genes in the genetic background of the inbreds in this study may influence levels of maltose accumulation. Kernel maltose accumulation may represent the expression of a gene linked to se. The embryo rescue procedure described in Expt. 1 could be used to clarify further the relationship between se and maltose.

Significantly lower kernel dry weight was observed in lightyellow kernels than in the dark-yellow kernels (Table 2, Fig. 3 ). Light-yellow kernels weighed $21 \%$ less than dark-yellow kernels. The $95 \%$ confidence ellipses contain the majority of kernels classified as either light or dark yellow with respect to sucrose and kernel dry weight (Fig. 3). The apparent cause of low kernel dry weight is not readily identifiable. Warm et al. (1980) identified differences in dry kernel weights and weights

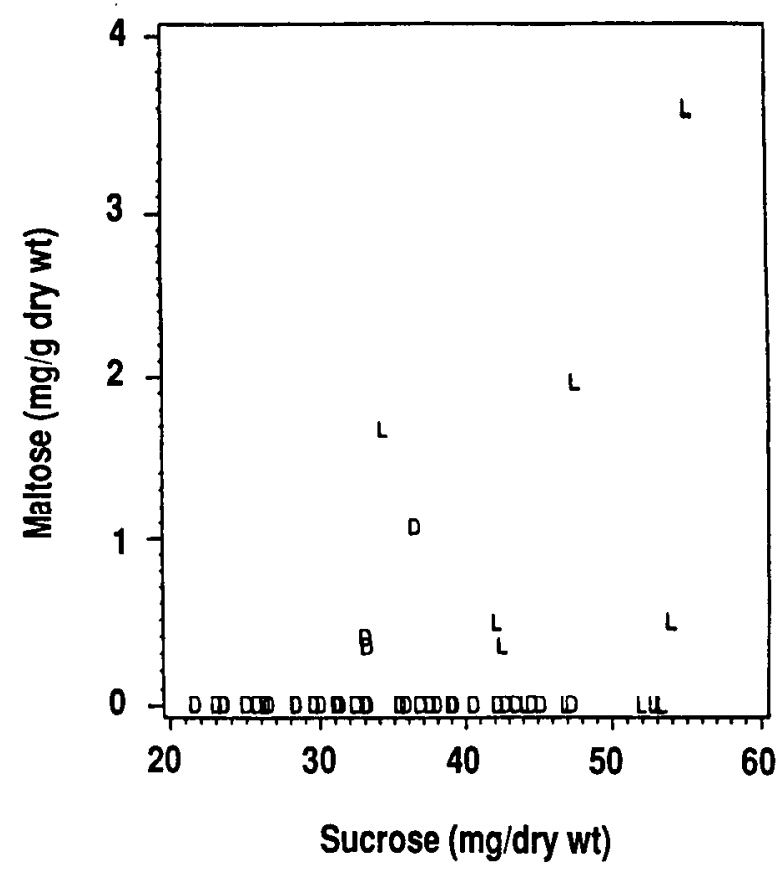

Fig. 2. Distribution of individual mature-dry seeds with respect to maltose and sucrose for $F_{2}$ kernels from a population (IL677a $\mathrm{X}$ IL451b)self, segregating for the se gene. $\mathrm{L}=$ light-yellow kernels, $\mathrm{D}=$ dark-yellow kernels.

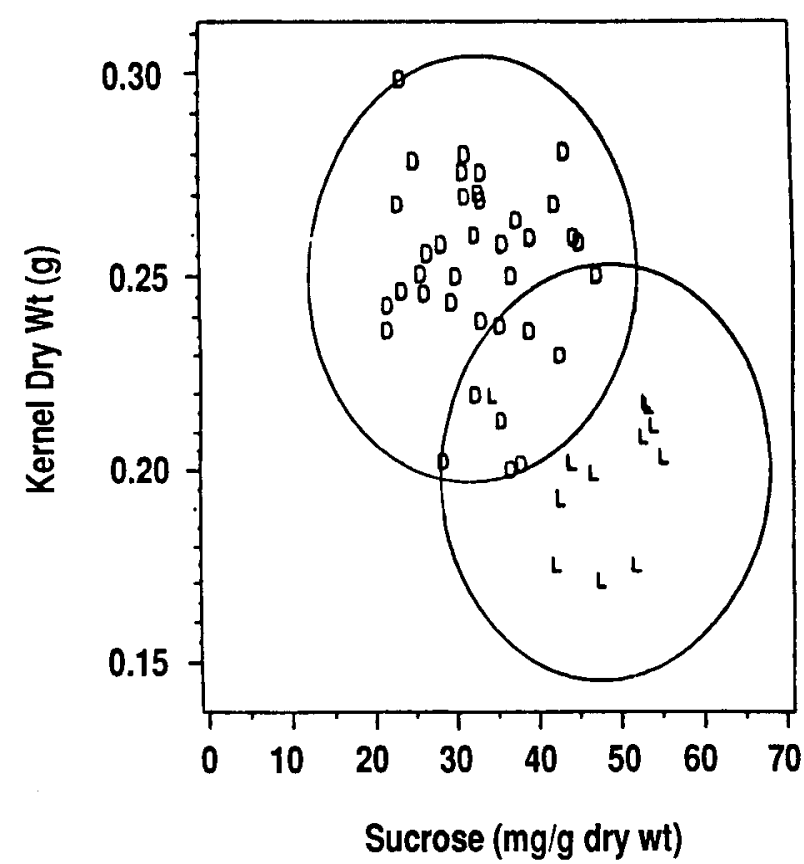

Fig. 3. Distribution of individual mature-dry seeds with respect to kernel dry weight and sucrose for $F_{2}$ kernels from a population (IL677a $x$ IL678a)self, segregating for the se gene. $\mathrm{L}=$ light-yellow kernels, $\mathrm{D}=$ dark-yellow kernels.

of both embryos and endosperm in comparisons of $S U-1$ and super sweet cultivars $(s h-2$, ae $d u w x)$. Super sweet cultivars tended to have lower kernel dry weights, endosperm, and embryo dry weights. The se gene, to a certain degree, decreased kernel starch content (Gonzales et al., 1976). Further investigations are required to substantiate this result. Breeders could use kernel dry weight as a means of selecting $s u-1$ se kernels, but poor seed vigor has also been associated with low kernel dry weight (Warm, 1980).

Moisture content. A significantly higher percentage of moisture was observed in light-yellow than in dark-yellow kernels (Table 2). Regression analysis indicates two distinct slopes fitting a linear regression model for kernels that are light- and dark-yellow (Fig. 4). Ferguson et al. (1979) suggested that moisture retention is an osmotic effect associated with the high levels of soluble carbohydrates (sugars and phytoglycogen) found in kernels homozygous for se. The concomitant increase in percent moisture content with sucrose observed in these data strengthens this hypothesis. Although the majority of kernels follow this trend, several kernels are relatively low in sucrose and high in percent moisture. These kernels could have compensated for lower sucrose by having higher levels of phytoglycogen as a result of expression of genes other than se in the segregating population.

In conclusion, individual su-1 se kernels in segregating populations can be identified based on metabolic and morphological traits. The sucrose content of a kernel is the direct phenotypic expression of se and, as such, provides the most reliable means of identifying su-1 se kernels. In contrast, elevated kernel maltose content is not always associated with enhanced sugar levels. Selection based on costly and time-consuming sugar analysis is impractical for breeders. A preferential means of identifying $s u$ $1 \mathrm{se}$ kernels is their light-yellow appearance. Selection of $s u-1$ se kernels based on moisture content (differential dry-down of 


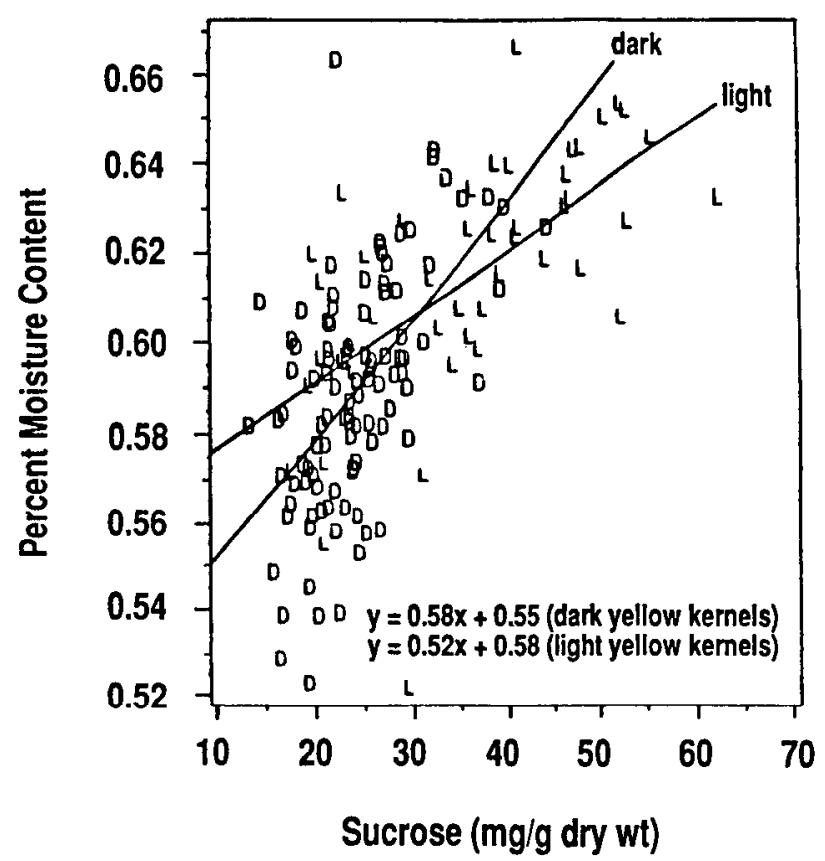

Fig. 4. Distribution of individual kernels at 35 days after pollination with respect to percent moisture content and sucrose for $\mathrm{F}_{2}$ kernels from a population (IL677a $\times$ IL451b)self, segregating for the se gene.

kernels) at 35 or 42 DAP is also suitable, but requires an added step of marking kernels before harvest, whereas selection based on color can be made after harvest of mature-dry seed. Selection based on moisture content would be necessary if environmental or genetic effects diminish differences in color between su-1 se and su-1 Se kernels or a dark-yellow su-1 se line were developed and incorporated into breeding programs. The ability to identify individual $s u-1$ se kernels in segregating populations has been facilitated by mapping of the chromosomal location of the $s e$ gene (La Bonte and Juvik, 1987b). Using the phenotypic selection criteria described above in conjunction with maize B-A translocation stocks (Beckett, 1976) these gene has been mapped in the maize nuclear genome (unpublished data). This step is important in the development of a genetic marker that could more readily identify $s u-1$ se kernels.

\section{Literature Cited}

Association of Official Analytical Chemists. 1984. Official methods of analysis. 13th ed. Assoc. Offic. Anal. Chem., Washington, D.C. Beckett, J.B. 1976. B-A translocations in maize. J. Hered. 69:27-36.

Cameron, J.W. and J.H. Teas, 1954. Carbohydrate relationships in developing and mature endosperm of brittle and related maize genotypes. Amer. J. Bot. 41:50-55.

Carey, E.E., A.M. Rhodes, and D.B. Dickinson. 1982. Postharvest levels of sugars and sorbitol in sugary enhancer (su se) and sugary maize (su Se). HortScience 17:241-242.
Creech, R.G. 1965. Genetic control of carbohydrate synthesis in maize endosperm. Genetics 52:1175-1186.

Creech, R.G. 1968. Carbohydrate synthesis in maize. Adv. Agron. 20:275-322.

Davies, B.H. 1976., Carotenoids in the chemistry and biochemistry of plant pigment. 2nd ed. Academic, London.

Ferguson, J.E., A.M. Rhodes, and D.B. Dickinson. 1978. The genetics of sugary enhancer (se), an independent modifier of sweet corn $(s u)$. J. Hered. 69:377-380.

Ferguson, J.E., D.B. Dickinson, and A.M. Rhodes. 1979. Analysis of endosperm sugars in a sweet corn inbred (Illinois 677a) which contains the sugary enhancer (se) gene and comparison of se with other corn genotypes. Plant Physiol. 63:416-421.

Flora, L.F. and R.C. Wiley. 1974. Sweet corn aroma, chemical components and relative importance in the overall flavor response. J. Food Sci. 39:770-773.

Garwood, D.L., F.J. McArdle, S.F. Vanderslice, and J.C. Shannon. 1976. Postharvest carbohydrate transformations and processed quality of high sugar maize in genotypes. J. Amer. Soc. Hort. Sci. 101:400-404.

Gonzales, J.W., A.M. Rhodes, and D.B. Dickinson. 1974. A new inbred with high sugar content in sweet corn. HortScience 9:79-80.

Gonzales, J.W., A.M. Rhodes, and D.B. Dickinson. 1976. Carbohydrate and enzymatic characterization of a high sucrose sugary inbred line of sweet corn. Plant Physiol. 58:28-32.

Green, C.E. and R.L. Phillips. 1975. Plant regeneration from tissue cultures of maize. Crop Sci. 15:417-421.

Juvik, J.A. and D.R. La Bonte, 1988. Single-kernel analysis for the presence of the sugary enhancer (se) gene in sweet corn. HortScience 23:384-386.

La Bonte, D.R. 1988. Clarification of the genetic nature of sugary enhancer, and endosperm character in sweet corn. PhD Diss., Univ. of Illinois, Urbana-Champaign.

La Bonte, D.R. and J.A. Juvik. 1987a. The expression of the sugary enhancer (se) gene in a starchy $(\mathrm{Su})$ background. Proc. of N. Central Reg. of ASHS 6th Annu. Mtg. July 1987. Brookings, S.D.

La Bonte, D.R. and J.A. Juvik. 1987b. The chromosomal location of the sugary enhancer (se) gene. Hort Science 22:136 (Abstr.).

Laughnan, J.R. 1953. The effect of sh 2 factor on carbohydrate reserves in the mature endosperm of maize. Genetics 38:485-499.

Lee, C.Y., P.E. McCoon and J.M. LeBowitz. 1981. Vitamin A value of sweet corn. J. Agr. Food Chem. 29: 1294-1295.

Linsmaier, E.M. and F. Skoog. 1965. Organic growth factor requirements of tobacco tissue cultures. Physiol. Plant 18:100-127.

Robertson, D.S., I.L. Anderson and M.D. Bachman. 1978. Pigment deficient mutants: genetic, biochemical, and developmental studies, p. 461-494. In: David B. Walton (ed.). Maize breeding and genetics. Wiley, New York.

Shannon, J.C. and D.L. Garwood. 1984. Genetics and physiology of starch, p. 25-86. In: R.L. Whistler, E.F. Paschall, and J.N. BeMiller (eds.). Starch: Chemistry and industry. 2nd ed. Academic, New York.

Soberlaske, R.M. and R.H. Andrew. 1978. Gene effects on kernel moisture and sugar of near isogenic lines of sweet corn. Crop Sci. 18:743-746.

Warm, E.V., G.B. Brown, and W.A. Hills. 1971. Genetic modification of sweet corn quality. J. Amer, Soc. Hort. Sci. 96:441-444.

Warm, E.V. 1980. Seed vigor and respiration of maize kernels with different endosperm genotypes. J. Amer. Soc. Hort. Sci. 105:3134. 